\title{
Philosophische Reflexion zu den Spielarten kollektiver Furcht
}

\begin{abstract}
Im Rahmen des Artikels wird der aktuell in zahlreichen Gesellschaftsanalysen bemühte Begriff der kollektiven Furcht einer genaueren Betrachtung unterzogen. Dabei erfahren drei Aspekte besondere Berücksichtigung: erstens das Verhältnis zwischen Individuum und Gruppe, zweitens die Funktion, die kollektive Furcht erfüllt, und drittens ihr destruktives Potential. Die genauere Analyse dieser Aspekte zeigt, dass wir mindestens drei verschiedene Formen von kollektiver Furcht unterscheiden sollten, die sich hinsichtlich dieser drei Dimensionen deutlich voneinander unterscheiden: ich-bezogene Furcht, Furcht aufgrund geteilter Anliegen und Furcht aufgrund von Identifikation mit der Gruppe. Wir haben es folglich hier nicht mit einem einheitlichen Phänomen zu tun, sondern vielmehr mit einer ganzen Reihe von zum Teil recht unterschiedlichen Erscheinungen.
\end{abstract}

The aim of this paper is to provide a thorough discussion of the term "collective fear" that is frequently used in current-day social analysis. Three aspects will be in focus: the relationship between the individual and the collective, the function of collective fear and its destructive potential. An analysis of these aspects shows that we should differentiate between at least three types of collective fear: I-focussed fear, fear based on shared concerns and fear based on identification with the group. Consequently, we should assume that we are not dealing with a single phenomenon here, but rather with a group of phenomena, each of which displays different structural features.

\section{Einleitung}

Kaum ein Begriff wird in aktuellen Gesellschaftsanalysen so häufig bemüht wie der der „kollektiven Furcht“. So spricht etwa der Soziologe Heinz Bude davon, dass wir in einer „Gesellschaft der Angst“ leben würden (Bude 2016), die Philosophin Martha Nussbaum warnt in ihrem jüngsten Werk davor, dass die Vereinigten Staaten zu einem „Königreich der Angst“ (Monarchy of Fear) zu werden drohen (Nussbaum 2019), der Historiker Frank Biess rekonstruiert in seinem Werk „Die Republik der Angst“ die bundesdeutsche Geschichte als die einer steten Abfolge unterschiedlicher kollektiver Furchtzustände (Biess 2019) und 
der Sozialwissenschaftler Oliver Nachtwey erklärt, dass wir es derzeit mit einer gesellschaftlichen Formation zu tun hätten, in der „die kollektive Angst vor dem sozialen Abstieg allgegenwärtig erscheint“ (Nachtwey 2017: 7). ${ }^{1}$

Ohne Zweifel helfen uns viele dieser Analysen, aktuelle Umbrüche und gesellschaftliche Herausforderungen besser $\mathrm{zu}$ begreifen. Allerdings bringt die Flut von Veröffentlichungen, die sich oberflächlich betrachtet eines sehr ähnlichen Begriffsvokabulars bedienen, auch gewisse Gefahren mit sich. Zu schnell verliert man, trotz aller begrifflicher Differenzierungen seitens der genannten Autoren, aus den Augen, dass wir es bei kollektiver Furcht nicht unbedingt mit nur einem einzigen Phänomen zu tun haben. Vielmehr könnte der Begriff durchaus auch verschiedene Phänomene bezeichnen, die sich möglicherweise in ihren gesellschaftlichen Auswirkungen deutlich voneinander unterscheiden.

Ausgehend von dieser Beobachtung möchte ich im Rahmen dieses Artikels drei verschiedene Spielarten kollektiver Furcht voneinander abgrenzen. Mir geht es dabei insbesondere um folgende Aspekte: Erstens soll das Verhältnis von Individuum und Gruppe in den Blick genommen werden. Hier ergeben sich eklatante Unterschiede zwischen den verschiedenen analysierten Formen kollektiver Furcht. Das heißt, die betrachteten Spielarten des kollektiven Fühlens sind tatsächlich jeweils in einem etwas anderen Sinne „kollektiv“.

Zweitens möchte ich die Funktion, die kollektiver Furcht jeweils zugeschrieben werden kann, etwas genauer betrachten. Dabei soll vor allem diskutiert werden, welche Rolle diese Emotion für den Aufbau und Erhalt von Gruppen spielt. Wie wir sehen werden, kommt zwar einigen Formen kollektiver Furcht eine stabilisierende Rolle zu. Das ist aber nicht bei allen hier besprochenen Typen der Fall.

Drittens scheint es mir auch wegen der bereits erwähnten zentralen Rolle, die kollektiver Furcht innerhalb der aktuellen Gesellschaftsanalyse zukommt,

1 In der philosophischen Debatte wird oft entweder im Rückgriff auf die Alltagssprache oder auch auf die existenzphilosophische Tradition zwischen Angst und Furcht unterschieden. Der Ausdruck ,Angst' soll dabei in der Regel eine ungerichtete Stimmung bezeichnen, während ,Furcht' eine Emotion bezeichnet, die sich auf ein konkretes Objekt (vor dem man sich fürchtet) richtet. Ich werde im Rahmen meines Artikels diese Unterscheidung beiseitelassen und die beiden Ausdrücke weitgehend Synonym verwenden. Diese Verwendung scheint mir einerseits legitim, weil ich mich hier ausschließlich mit Fällen beschäftigen werde, in denen konkrete Objekte eine Rolle spielen. Es besteht deshalb nicht die Gefahr, zwei strukturell verschiedene Phänomene miteinander zu verwechseln. Zweitens zeigt die aktuelle Forschungsliteratur, dass die gängige Unterscheidung zwischen Furcht und Angst nicht so gut begründet ist, wie man auf den ersten Blick annehmen könnte. Für eine ausführliche Diskussion des Verhältnisses vgl. Tietjen (2019). 
geboten, dem destruktiven Potential dieser Emotion besondere Aufmerksamkeit zu schenken. Ausgangspunkt ist die These Martha Nussbaums, dass Furcht zwar evolutionär gesprochen eine sehr wertvolle Emotion ist, dass sie aber im Bereich der Politik schnell zu problematischen Konsequenzen führen kann (Nussbaum 2019). Diese Analyse ist im Kern sicherlich richtig, aber sie bedarf aus meiner Sicht der weiteren Differenzierung. So werde ich im Rahmen des Artikels auch aufzeigen, dass jede der besprochenen Spielarten kollektiver Furcht ein jeweils etwas anders zu charakterisierendes destruktives Potential in sich birgt. Erst wenn wir uns über die Unterschiede zwischen diesen verschiedenen Spielarten im Klaren sind, können wir also die Gefahren genauer fassen und erkennen, die (neben allen potentiellen positiven Effekten) von kollektiven Furchtzuständen ausgehen.

\section{Furcht und Werturteile}

Bevor ich auf die unterschiedlichen Spielarten kollektiver Furcht eingehe, möchte ich zunächst eine weitere wichtige Abgrenzung vornehmen. Geht man von unserer Alltagssprache aus, dann fällt auf, dass gerade im medialen Bereich häufig großen Kollektiven Furcht zugeschrieben wird. So titelte beispielsweise unlängst der Spiegel „Britische Universitäten fürchten den ungeregelten Brexit“ (Klovert 2019).

Solche Furchtzuschreibungen sind nicht ungewöhnlich. Trotzdem muss man genau untersuchen, ob es sich bei den zugeschriebenen Zuständen wirklich um Furcht in einem vollwertigen Sinne handelt. Häufig scheinen die jeweiligen Autoren nämlich einen rein kognitiven Zustand beschreiben zu wollen. So ist vermutlich die Autorin des Spiegel-Artikels nicht der Ansicht, dass die britischen Universitäten (bzw. die Leitung dieser Universitäten) angesichts des Brexits in starke Furchtzustände versetzt worden sind. Plausibler scheint, dass hier der Umstand beschrieben werden soll, dass diese Personengruppe einen ungeregelten Brexit für möglich hält und weiterhin der Ansicht ist, dass dieser äußerst negative Konsequenzen hätte. Es handelt sich also zunächst um eine in einer bestimmten Gruppe breit geteilte Einschätzung der Situation. Man könnte auch sagen, dass in diesem Fall ein bestimmtes Urteil von den Vertretern der Gruppe getroffen wird. Dieses ließe sich beispielsweise wie folgt ausdrücken: „Es ist nicht ausgeschlossen, dass der ungeregelte Brexit eintritt, und dieser wäre für die Finanzierungslage britischer Hochschulen bedrohlich.“

Nun stellt sich die Frage, ob eine solche Einschätzung bzw. ein solches Urteil bereits als Emotion zu werten ist. Tatsächlich mag es auf den ersten Blick so 
scheinen, als würde eine solche Ansicht in der aktuellen Emotionstheorie tatsächlich vertreten. So haben sowohl Martha Nussbaum wie auch Robert Solomon dafür argumentiert, Emotionen als eine spezifische Form von Werturteilen aufzufassen (Nussbaum 2001; Solomon 1993). Furcht besteht diesen Autoren zufolge wesentlich darin, ein Objekt als bedrohlich oder gefährlich zu beurteilen. Allerdings ist auch unter Voraussetzung dieser Emotionstheorien fraglich, ob wir bei dem Beispiel von einer Emotion im vollwertigen Sinne sprechen können. Schließlich würden auch diese Autoren nicht behaupten wollen, dass jede Form von Werturteil schon mit einer Emotion gleichzusetzen ist. Vielmehr nehmen diese Philosophen an, dass Emotionen affektive Werturteile darstellen. Was aber zeichnet solche affektiven Urteile gegenüber nicht-affektiven aus?

Eine mögliche Antwort bestünde hier darin, auf die Erlebnisqualitäten zu verweisen, die gemeinhin mit Emotionen einhergehen. Es fühlt sich schließlich auf bestimmte Art und Weise an, Furcht zu empfinden, während dies bei rein kognitiven Urteilen auf den ersten Blick nicht der Fall zu sein scheint. ${ }^{2}$

Das bedeutet, dass die Frage, ob wir es bei dem zugeschriebenen Zustand mit Furcht im engeren Sinne zu tun haben, davon abhängt, ob die Gruppe (bzw. die Individuen, die sie konstituieren) in einem Zustand mit der für Furcht typischen Erlebnisqualität sind. Sind also die fraglichen Personen in einem Zustand der Erregung, haben sie beispielsweise einen beschleunigten Puls oder das Gefühl, dass ihre Gedanken hin und her rasen? Oder fühlen sie, wie ihre Muskeln sich anspannen? Bei dem hier angeführten Beispiel ist das wahrscheinlich kaum der Fall. Vermutlich möchte die Autorin des Artikels den Vertretern britischer Universitäten einen solchen affektiven Zustand gar nicht zuschreiben. Plausibler ist, dass sie den Ausdruck „Furcht“ in einem sehr weiten Sinne verwendet. Bezeichnet werden sollen vermutlich die entsprechenden Werturteile, aber nicht die furchttypischen phänomenalen Qualitäten. ${ }^{3}$

2 Auch wenn Nussbaum und Solomon durchaus bestimmte phänomenale Komponenten von Emotionen integrieren wollen, so ist doch umstritten ob ihr theoretischen Ansatz dies überhaupt erlaubt. Kritische Positionen sind diesbezüglich unter anderem von Robinson und Roberts vertreten worden: Vgl. Robinson (2005); Roberts (2003).

3 Mit meiner Betonung der Bedeutung von phänomenalen Qualitäten für Emotionen geht zudem auch eine ontologische Festlegung einher. Wenn es entscheidend für die Zuschreibung von Emotionen ist, dass wir die entsprechenden Empfindungsqualitäten aufweisen, dann können wir strikt genommen nur Individuen Emotionen zuschreiben. Damit schließe ich für den Rahmen dieses Artikels Konzeptionen von Gruppenemotionen aus, die ein sehr starkes, ontologisches Verständnis von Kollektivität zugrunde legen. Vertreter solcher Theorien gehen davon aus, dass nur diejenigen Emotionen wirklich als „kollektiv“ zu bezeichnen sind, die auf Gruppenebene vorliegen. So hat Bryce Huebner etwa die Behauptung aufgestellt, wir könnten 
Die Fälle, in denen es nur um Furcht in diesem sehr weit gefassten Sinne geht, möchte ich im Folgenden ausklammern. Mich interessieren im Weiteren also ausschließlich Fälle kollektiver Furcht, bei denen auch die phänomenale Komponente vorliegt und wir es folglich nicht (nur) mit einem bestimmten Werturteil zu tun haben.

\section{Ich-bezogene Furcht und Gruppen als Zweckgemeinschaften}

Betrachten wir nun also die Rolle, die Furcht in diesem engeren Sinne innerhalb von Gruppen spielen kann. Dass Furcht in diesem Sinne überhaupt irgendeine positive Relevanz für Gruppen haben kann, ist alles andere als selbstverständlich. Furcht ist kein genuin sozialer Affekt. So kann sie auch bei Subjekten auftreten, die in keine gesellschaftliche Struktur eingebunden sind. Darin unterscheidet sie sich klar von anderen emotionalen Reaktionen wie etwa Schamgefühlen, die nur in sozialen Kontexten auftreten können.

Furcht scheint aber noch aus einem anderen Grund prima facie nicht recht in den Bereich des Sozialen passen zu wollen. Furcht in seiner prototypischen Ausprägung ist zunächst einmal Furcht um das eigene Leben bzw. das eigene körperliche Wohlergehen. Man könnte dementsprechend auch sagen, dass es sich zunächst einmal um einen zutiefst egozentrischen Affekt handelt. Furcht in ihrer basalsten Ausprägung wirft uns zurück auf unser ureigenstes Interesse, nämlich das Interesse, in einer oftmals feindlichen Welt $\mathrm{zu}$ überleben. Sie fokussiert uns deshalb darauf, unser eigenes Überleben zu sichern, koste es, was es wolle. Natürlich gibt es Fälle, in denen sich Menschen auch in Gefahrensituationen altruistisch verhalten, aber wir sollten uns darüber im Klaren sein, dass sie dies meist trotz ihrer Furcht tun und nicht aufgrund ihres emotionalen $\mathrm{Zu}$ stands. ${ }^{4}$

auch dann von der kollektiven Furcht einer Gruppe reden, wenn diese furchttypische Situationseinschätzungen sowie furchttypisches Verhalten an den Tag legt. Das ist seiner Ansicht nach selbst dann möglich, wenn kein Individuum innerhalb der Gruppe (sondern eben nur die Gruppe in ihrer Gesamtheit) Furcht aufweist (vgl. Huebner (2011)). Eine solche Konzeption kollektiver Emotionen schließt jedoch notgedrungen die von mir betonten phänomenalen Komponenten aus.

4 Auf diesen Aspekt weist auch Martha Nussbaum hin: „Angst ist in der Tat sehr narzisstisch. Sie vertreibt alle Gedanken an andere, selbst wenn diese bereits in irgendeiner Form in uns verwurzelt sind. Die Angst eines Säuglings konzentriert sich vollständig auf seinen eigenen 
Die egozentrischen Aspekte dieser prototypischen Fälle von Furcht scheinen uns auch in unserer theoretischen Betrachtung zunächst gänzlich auf das Individuum zurückzuwerfen. Das muss aber nicht folgen. Furcht um das eigene Leben kann uns schließlich auch dazu motivieren, in eine bestimmte Gruppe einzutreten, sofern die Mitgliedschaft in diesem Kollektiv das eigene Überleben wahrscheinlicher macht. In anderen Worten kann uns Furcht gerade auch aufgrund ihrer egozentrischen Aspekte in die Arme der Gesellschaft treiben. ${ }^{5}$ Das nährt wiederum die Vermutung, dass Furcht tatsächlich eine zentrale Rolle bei der Ausbildung von Gruppen spielen kann.

Die gruppenkonstituierende Rolle von ich-bezogener Furcht ist nicht nur auf Fälle beschränkt, in denen das Leben des Individuums unmittelbar bedroht ist. Vielmehr kann dergleichen auch in Fällen auftreten, in denen ein Individuum ein Gut als bedroht ansieht, dem es einen zentralen Stellenwert in seinem Leben zumisst. Ein gutes Beispiel für einen solchen Fall sind die bereits zu Beginn des Artikels erwähnten Abstiegsängste, die ich nun vor dem Hintergrund des bislang Erarbeiteten einer genaueren Analyse unterziehen möchte.

Abstiegsängste mögen in einer Gesellschaft weit verbreitet sein, in einem strikten Sinne sind sie jedoch fast nie „kollektiv“. Vielmehr zeigt sich in ihnen die bereits erwähnte klassische Ich-Fokussierung existentieller Angst. Wenn ich unter Abstiegsängsten leide, dann fürchte ich mich zunächst einmal davor, dass ich sozial absteige, etwa weil ich meinen Arbeitsplatz verliere. Der soziale Abstieg anderer spielt in meinen Überlegungen hingegen kaum eine Rolle. Es ist also zumeist nicht so, dass hier eine Gruppe von Individuen fürchtet, gemeinsam abzusteigen, so wie etwa eine Fußballmannschaft den Abstieg in die Zweite Bundesliga fürchten mag.

Trotzdem ist es natürlich nicht falsch, diese Ängste als „kollektiv“ (in einem weiter gefassten Sinne) zu bezeichnen. Auch wenn die Angst jedes einzelnen sich auf ein anderes Objekt richtet (nämlich den jeweils eigenen sozialen Abstieg), so ist es doch denkbar, dass Abstiegsängste als Emotionstyp in bestimmten sozialen Kontexten dominant werden können. So können solche Ängste eine zentrale Rolle einnehmen, wenn beispielsweise innerhalb einer Gesell-

Körper. Selbst wenn wir später in der Lage sind, uns um andere zu sorgen, treibt die Angst diese Sorge oft aus und wirft uns auf die Stufe eines kindlichen Solipsismus zurück“ (Nussbaum 2019: 49).

5 Der Gedanke, dass Furcht uns zum Eintritt in die Gesellschaft motivieren kann, ist in seiner prägnantesten Form wohl von Thomas Hobbes formuliert worden (vgl. Hobbes 1996). Allerdings bezieht sich Hobbes nur zum Teil auf den sehr basalen Furchtbegriff, den ich hier skizziert habe. Für eine aktuelle politikwissenschaftliche Analyse dieser Position, die auch der Komplexität des Furchtbegriffs bei Hobbes Rechnung trägt, vgl. Corey (2006). 
schaft oder sozialen Gruppe ein hoher Prozentsatz von Individuen solche Ängste aufweist. ${ }^{6}$

Ängsten dieser Art kann zudem eine wichtige gruppenbildende Funktion zukommen. Meine Angst vor dem drohenden sozialen Abstieg kann mich dazu motivieren, mich mit anderen zusammenzutun, die ebenfalls unter Abstiegsängsten leiden. Diese Motivation ist insbesondere dann gegeben, wenn es erfolgsversprechend erscheint, die Gefahrenquelle gemeinsam zu bekämpfen. So könnte es beispielsweise sinnvoll erscheinen, gemeinsam gegen bestimmte staatliche Kürzungen bei den Sozialausgaben zu demonstrieren. ${ }^{7}$ Wir sehen hier wieder, wie tatsächlich auch strikt genommen rein ich-bezogene Emotionen uns dennoch zur Gründung einer Gruppe bzw. zum Beitritt in eine bereits bestehende Gruppe motivieren können.

Allerdings haben wir es hier nur mit einer sehr eingeschränkten Einbindung von Individuen in Gruppen zu tun. Es ist schließlich nicht so, als wäre mit dem Zusammenschluss vieler furchterfüllter Individuen zu einer größeren Gruppe die gänzlich auf das einzelne Individuum konzentrierte Furcht aus der Welt geschafft. Diese besteht vielmehr als individuelle Angst auch in der nun begründeten Gruppe weiter fort. Furcht motiviert das Individuum, in die Gruppe einzutreten, aber sie kann es auch dazu motivieren, die Gruppe wieder zu verlassen. Das geschieht dann, wenn das Individuum seine Interessen in dieser Gruppe nicht optimal vertreten sieht. Der Eindruck, dass die individuellen Belange nicht ausreichend berücksichtigt werden, kann vielfältige Gründe haben. Betrachten wir dazu noch einmal den Fall der Abstiegsängste. Hier kann beispielsweise der Eindruck entstehen, dass sich die Gruppe nicht genug für den Erhalt des eigenen Arbeitsplatzes einsetzt, sondern andere Gruppenmitglieder (die vielleicht in anderen Branchen tätig sind) privilegiert. Oder das Individuum kann erkennen, dass es deutlich weniger abstiegsgefährdet ist, als zunächst gedacht, und deshalb die Gruppe verlassen (da sie ja für die eigenen Belange nicht mehr von Bedeutung ist). Was wir hier also sehen, ist, dass die resultierenden Gruppen nur solange stabil sind, wie sie den jeweiligen Einzelinteressen der Mitglieder optimal dienen. Auf das Beispiel zurückbezogen bedeutet das:

6 Diese Emotionen wären also in einem sehr schwachen aggregativen Sinne als kollektiv zu bezeichnen. Allein der Umstand, dass die Mehrheit in einer Gruppe solche Ängste aufweist, macht sie schon zu einem als kollektiv zu klassifizierenden Phänomen. Ein (allerdings sehr viel ausgefeilteres) aggregatives Modell kollektiver mentaler Zustände ist in den letzten Jahren insbesondere von Pettit und List vertreten worden. Vgl. List \& Pettit (2011).

7 Auf diese Aspekte weist auch Salmela hin, der diese Emotionstypen als schwach kollektiv klassifiziert und ebenfalls darauf hinweist, dass diese dennoch die Basis kooperativer Unterfangen bilden können. Vgl. Salmela (2014: 170). 
solange die Gruppe den Abstieg jedes einzelnen Gruppenmitglieds unwahrscheinlicher macht. Sobald die Gruppe diese Funktion nicht mehr für alle Mitglieder erfüllen kann, ist damit zu rechnen, dass sie auch nicht in ihrer jetzigen Form weiter besteht, weil die nicht-ausreichend repräsentierten Mitglieder die Gruppe verlassen oder umformen werden.

Hier wird eine zentrale strukturelle Eigenschaft der beschriebenen Situation deutlich: Die Gruppe hat in diesem Fall keinen eigenen Wert für die Gruppenmitglieder. Sie ist lediglich Mittel zum Zweck. Ihre einzige Daseinsberechtigung besteht darin, dass sie den einzelnen Gruppenmitgliedern erlaubt, ihre jeweils eigenen Ziele besser durchzusetzen.

Zusammenfassend können wir also festhalten, dass ich-bezogene Furcht ein Affekt ist, der uns zunächst einmal auf unsere eigenen Interessen und vor allem unser basalstes Interesse, nämlich das eigene Überleben, zurückwirft. Trotz dieser scheinbar „unsozialen“ Komponente kann auch dieser Affekt durchaus zu Gruppenbildungen beitragen. Allerdings sind die so geformten Gruppen keine sehr stabilen Gebilde. Sie erlauben die Koordination von Handlungen, aber zerfallen, wenn sie den einzelnen Mitgliedern nicht mehr zur Erreichung ihrer eigenen Ziele dienlich sind.

In ihrer exklusiven Fokussierung auf das Individuum liegt auch das destruktive Potential dieser Form von kollektiver Furcht begründet. Auch wenn wir unsere Handlungen innerhalb der Gruppe koordinieren, so bleibt doch in diesem Rahmen kein Raum, die Interessen anderer zu berücksichtigen. Diese Interessen fließen nur dann ein, wenn sie für das Erreichen der eigenen Ziele relevant sind. In einer von solchen ich-bezogenen Ängsten dominierten Gruppe scheinen deshalb stabile Beziehungen, die sich gerade auch durch ein nicht von eigenen Interessen geleiteten Eintreten für den anderen auszeichnen, nur schwer vorstellbar. Massive Furcht scheint diese Beziehungen eher zu unterminieren, als sie zu stützen, und gerade darin liegt das destruktive Potential dieses Affekts.

\section{Geteilte Anliegen und kollektive Furcht}

Ich habe mich bisher auf prototypische Fälle von Furcht konzentriert, in denen das Individuum sich selbst als bedroht erfährt. Das ist aber nicht die einzige Form, die Furcht annehmen kann. Vielmehr kann Furcht auch dort auftreten, wo die Bedrohung eine weniger existentielle Form annimmt. So kann ich beispielsweise als Hobby-Gärtner angesichts einer bevorstehenden Frostperiode um die von mir angepflanzten Tomaten fürchten, obwohl von diesem Ereignis 
keine unmittelbare Bedrohung für mich ausgeht. Vielmehr ist hier lediglich etwas gefährdet, das für mich einen gewissen Wert hat, ohne dass ich selbst deshalb schon einer Gefahr ausgesetzt wäre. ${ }^{8}$

Interessant ist dieses Beispiel, weil es darauf verweist, dass ich als Individuum bestimmte Anliegen habe und dass diese Anliegen sich auch auf mein emotionales Erleben auswirken. Wäre das Gärtnern für mich nur eine lästige Pflicht, der ich mich am liebsten entledigen würde, dann würde ich in der besagten Situation vermutlich nicht mit Furcht reagieren. In der philosophischen Literatur zu Emotionen hat sich dementsprechend auch der Gedanke durchgesetzt, dass emotionale Reaktionen ihre Grundlage vielfach in bestimmten Anliegen von Individuen haben. ${ }^{9}$

Anliegen können hochgradig individuell sein. So ist vorstellbar, dass niemand sonst meine Liebe zum Gärtnern teilt. Gleichermaßen ist aber auch vorstellbar, dass ich ein bestimmtes Anliegen mit anderen teile. Man könnte in diesem Zusammenhang beispielsweise an eine Umweltschutzgruppe denken. Allen Mitgliedern dieser Gruppe ist gemein, dass ihnen der Schutz und Erhalt der Umwelt am Herzen liegt. Weiterhin können die Mitglieder auch wissen, dass das nicht einfach nur ihr persönliches Anliegen ist. Vielmehr kann ihnen durchaus bewusst sein, dass ihre Gruppe durch ein gemeinsames Anliegen geeint wird, bzw. dass dieses Anliegen etwas ist, das sie mit anderen teilen. So ist den Umweltaktivisten beispielsweise allen gleichermaßen der Schutz des Regenwaldes wichtig. Ein solches geteiltes Anliegen kann zugleich auch die Basis einer kollektiven emotionalen Reaktion darstellen. So können die Mitglieder der Gruppe mit Furcht reagieren, wenn sie erfahren, dass ein Teil des Regenwaldes abgeholzt werden soll. ${ }^{10}$

8 Das Beispiel übernehme ich von Bennett Helm: Helm (2009).

9 Als prominenteste Vertreter dieser Ansicht sind insbesondere Helm und Roberts zu nennen: Vgl. Helm (2001); Roberts (2003). Beide plädieren im Kern für eine rationale Beziehung zwischen Anliegen und Emotion. Ich selbst habe mich dafür ausgesprochen, die Beziehung lediglich als kausal zu verstehen. Ein bestimmtes Anliegen ist dieser Konzeption zufolge lediglich als eine mögliche Ursache einer bestimmten emotionalen Reaktion zu begreifen (vgl. Berninger (2017)). Für die Überlegungen, die ich in diesem Artikel zu kollektiven Emotionen anstellen möchte, ist die Unterscheidung jedoch nebensächlich. Dementsprechend gehe ich an dieser Stelle über diese Differenzen hinweg.

10 Die Vorstellung, dass geteilte Anliegen die Basis kollektiver Emotionen darstellen, ist sowohl von Helm wie auch von Salmela in die Debatte eingebracht worden: Vgl. Helm (2008); Salmela (2014). 
Anliegen müssen nicht völlig deckungsgleich sein, um geteilt $\mathrm{zu}$ werden. Vielmehr erlaubt die Konzeption hier durchaus auch graduelle Abstufungen. ${ }^{11}$ So kann einem Teil der Umweltschützer vor allem das Klima am Herzen liegen, während anderen wiederum der Erhalt gefährdeter Tierarten besonders wichtig ist. Die unterschiedlichen Vertreter der Gruppe können sich sogar ihrer Differenzen bewusst sein, aber dennoch das Anliegen, den Regenwald zu retten, als etwas Geteiltes erfahren, ebenso wie die auf diesem Anliegen basierende Furchtreaktion.

Die auf solchen geteilten Anliegen basierenden Formen von Furcht unterscheiden sich strukturell sehr deutlich von den im letzten Abschnitt beschriebenen ich-bezogenen Formen von Furcht. Zentral ist in diesem Fall, dass ich die Emotion als etwas erfahre, das ich aufgrund bestimmter geteilter Anliegen mit anderen ebenfalls teile. Die Emotion wird als etwas erlebt, das aufs engste mit bestimmten gemeinsamen Projekten zusammenhängt. Dementsprechend werde ich (als Teil einer Gruppe) eben auch nicht, wie bei der ich-bezogenen Furcht, auf eine rein egozentrische Perspektive zurückgeworfen. Es geht nicht primär um mich, sondern vielmehr um das mit anderen geteilte Anliegen (also in unserem Beispiel um das gemeinsame Anliegen, den Regenwald zu schützen). Es scheint plausibel, dass solche geteilten (und als geteilt erkannten) Anliegen wichtige Funktionen für Gruppen erfüllen. Einerseits werden viele Gruppen nur deshalb gegründet, weil Individuen erkennen, dass sie Anliegen teilen und diese Anliegen gemeinsam verfolgen wollen. Weiterhin bilden solche Anliegen einen wichtigen Kitt für bereits existierende Gruppen. So ist beispielsweise vorstellbar, dass ich mich über irgendeinen Vorfall innerhalb der bestehenden Gruppe zwar maßlos ärgere, aber dennoch in der Gruppe bleibe, weil mir das geteilte Anliegen nach wie vor viel bedeutet. Furcht, aber auch anderen geteilten Emotionen, kommt hier ebenfalls eine wichtige Rolle zu. Die gemeinsam empfundene und zum Ausdruck gebrachte Furcht kann uns als Gruppenmitgliedern verdeutlichen, dass wir bestimmte Emotionen teilen, weil uns ähnliche Dinge wichtig sind. Auch das kann gruppenstabilisierende Wirkung haben.

Es zeigt sich hier also, dass das destruktive Potential kollektiver Furcht in dem nun betrachteten Fall nicht darin liegt, dass sie uns einzig auf uns allein zurückwirft und soziale Beziehungen dadurch unterminiert. Ganz im Gegenteil scheint in diesem Fall der Bezugspunkt in der Gruppe selbst zu liegen. Furcht ist hier vielfach also nicht zersetzend, sondern kittend $\mathrm{zu}$ verstehen. Trotzdem weist auch diese Form von Furcht aus meiner Sicht eine nicht zu unterschätzende destruktive Komponente auf. Um zu sehen, worin diese besteht, muss

11 Auch auf diesen Aspekt hat bereits Salmela hingewiesen. Vgl. Salmela (2014). 
man aber einen weiteren Aspekt von Emotionen im Allgemeinen und Furcht im Besonderen in den Blick nehmen, den ich bislang beiseitegelassen habe. Furcht ist nicht nur ein Zustand mit einer bestimmten phänomenalen Komponente. Vielmehr gehen mit ihm zahlreiche kognitive Veränderungen einher. ${ }^{12}$ Ich will hier nur auf zwei für unsere Zwecke besonders wichtige Ergebnisse hinweisen. So hat Barbara Fredrickson aufgezeigt, dass positive Emotionen wie Freude zu einer Erweiterung des Handlungsrepertoires von Versuchspersonen führen, während Furcht und Wut es verengen (Fredrickson 2001). Das legt die Vermutung nahe, dass unter dem Einfluss von Furcht eher auf bekannte Handlungsmuster zurückgegriffen wird, während man unter dem Einfluss von positiven Emotionen eher bereit ist, neue, kreative Handlungsweisen zu erkunden. ${ }^{13}$ Weiterhin sprechen zumindest einige empirische Ergebnisse dafür, dass mit Furcht eine Fokussierung der Aufmerksamkeit auf potentielle Bedrohungen einhergeht (Quigley et al. 2012).

Diese kognitiven Veränderungen müssen nicht unbedingt negative Auswirkungen für das Subjekt haben. So kann es in gefährlichen Situationen evolutionär gesprochen durchaus sinnvoll sein, sich auf bereits bekannte, etablierte Handlungsmuster zurückzuziehen. Dennoch zeigt sich, dass Furcht, ganz unabhängig davon, ob sie individueller oder kollektiver Natur ist, problematische Aspekte mit sich bringt. So scheint Furcht in Situationen, die nach kreativen Problemlösungen verlangen, eher kontraproduktiv zu sein, weil sie den Rückgriff auf übliche Bewältigungsstrategien wahrscheinlicher macht.

Auch wenn solche Probleme gleichermaßen auf individueller Ebene auftauchen, so ist es plausibel, dass sie im Rahmen von Kollektiven noch weiter verschärft werden. Die zusätzliche Schwierigkeit bei Gruppen besteht darin, dass sich die einzelnen Gruppenmitglieder in ihrer (durch ihre Furcht begründeten) Fokussierung auf Bedrohungen und in ihrem eher rigiden, wenig kreativen Problemlösungsverhalten möglicherweise noch untereinander bestärken. Das destruktive Potential der Emotion besteht also darin, dass sich die Gruppe insgesamt zu sehr auf einzelne Aspekte einer Situation konzentriert, dadurch andere Eigenschaften aus dem Blick verliert und zudem wenig offen für ungewöhnlichere Problemlösungen ist.

12 Philosophisch gesprochen ist der Status dieser kognitiven Veränderungen umstritten. Ich habe dafür plädiert, ihn nicht als bloßen Effekt von Emotionen, sondern vielmehr als Teil der Emotion selbst aufzufassen. Vgl. Berninger (2017).

13 Vgl. dazu auch Isen, Daubman \& Nowicki (1987). Auch in den dort geschilderten Experimenten zeigte sich die besondere Bedeutung von positiven Emotionen für das kreative Problemlösungsverhalten der Probanden. 


\section{Kollektive Furcht auf Basis der Identifikation mit der Gruppe}

Wir haben bislang gesehen, dass geteilte Anliegen die Basis für Furchtreaktionen bilden können, die eben nicht die egozentrische Fokussierung prototypischer Furcht aufweisen. Der zentrale Schritt war dabei, dass das Individuum die fraglichen Anliegen nicht mehr nur als seine eigenen erfährt, sondern sie vielmehr als geteilt begreift. Man könnte deshalb vermuten, dass die mit Furcht verbundene egozentrische Tendenz dann nivelliert wird, wenn die Gruppe eine größere Rolle spielt, die Emotion also in mehr als nur einem schwachen Sinne als kollektiv zu bezeichnen ist. Dieser Eindruck täuscht jedoch, wie ich in diesem letzten Abschnitt aufzeigen möchte. So können auch bei solchen Furchtreaktionen, die wir als kollektiv in einem sehr starken Sinne beschreiben würden, Eigenschaften auftreten, die den zu Beginn beschriebenen egozentrischen Tendenzen sehr ähnlich sind. Wie ich zeigen werde, treten diese Aspekte insbesondere dann auf, wenn eine Identifikationsbeziehung zwischen Individuum und Gruppe vorliegt.

Betrachten wir also zunächst einmal genauer, worin eine solche Identifikationsbeziehung besteht. Was bedeutet es also, wenn wir sagen, dass ein Individuum sich mit einer bestimmten Gruppe identifiziert? Zunächst kann damit einfach nur gemeint sein, dass dieses Individuum in irgendeiner Form Anteil am Schicksal der Gruppe nimmt. In diesem Sinne kann man vielleicht sagen, dass sich ein Menschenrechtsaktivist mit den Rohinga identifiziert. ,Identifikation' heißt hier zunächst einmal nur: Dem Aktivisten ist es wichtig, wie es dieser Gruppe ergeht. Eine solche Identifikation kann durchaus auch die Grundlage einer emotionalen Reaktion sein, etwa wenn die Person sich um das Wohlergehen der Gruppe sorgt oder die unmittelbar bevorstehende Vertreibung der Rohinga aus Myanmar bei ihr Furcht auslöst.

Es gibt aber noch eine etwas anders gelagerte Form von Identifikationsbeziehung. Diese liegt dann vor, wenn sich jemand stark mit einer Gruppe identifiziert, deren Mitglied er oder sie ist. Eine solche Form von Identifikation liegt meist dann vor, wenn die fragliche Gruppe eine zentrale Rolle im eigenen Leben einnimmt. ${ }^{14}$ Betrachten wir dazu folgendes Beispiel: Nehmen wir an, Anna und Berta sind beide Mitglieder in einem Schachclub. Anna spielt zwar gut und gerne Schach, aber letztendlich ist das für sie dennoch nicht mehr als ein Hob-

14 Darauf, dass solche Identifikationsbeziehungen eine wichtige Rolle spielen können, verweisen u. a. auch von Scheve und Ismer. Vgl. von Scheve \& Ismer (2013). 
by. Der Schachclub ist ihr zwar wichtig, sie wäre traurig, wenn er wegen Mitgliederschwund schießen müsste, aber er nimmt dennoch keine zentrale Rolle in ihrem Leben ein. Bei Berta ist die Situation anders gelagert. Für sie ist Schach deutlich mehr als nur ein Hobby. Und auch der Schachclub nimmt für sie eine ganz andere Rolle ein: Sie verbringt dort jede Minute ihrer freien Zeit, sie verfolgt aufmerksam alle Clubnachrichten und ist auch bei allen Vereinstreffen dabei. Man könnte dementsprechend sagen, dass dieser Club für sie lebensbestimmend ist. Gäbe es den Club nicht mehr, würde sich Bertas Leben auf radikale Weise verändern. Ihre Mitgliedschaft im Schachclub ist für sie nicht einfach nur die Mitgliedschaft in einer Gruppe neben vielen anderen, zu denen sie auch noch gehört (wie das bei Anna vermutlich der Fall ist).

Weil Bertas Leben eng mit dem Schachclub verknüpft ist, hätte die SchlieBung desselben für sie auch viel weitreichendere Folgen als für Anna. Wenn es den Schachclub nicht mehr gäbe, müsste Berta ihr Leben ganz anders strukturieren als bisher. In diesem Sinne könnte man sagen, dass sie in diesem Fall selbst auch eine andere werden müsste. Ihr Leben ist so eng mit dem Schachclub verknüpft, dass jede Änderung auch auf sie selbst unmittelbare Auswirkungen hätte. Man kann dementsprechend sagen, dass die Gefährdung der Fortexistenz des Schachclubs zugleich auch Bertas Leben in seiner derzeitigen Struktur gefährden würde. Dementsprechend ist es auch verständlich, dass Berta auf eine mögliche Schließung des Schachclubs mit sehr viel stärkeren, tiefergehenden Emotionen reagieren wird als Anna.

In diesem Fall ist Bertas Angst äußerst eng an das Kollektiv gebunden. Sie empfindet diese Furcht nicht als einzelne, sondern vielmehr als Mitglied einer Gruppe. Darin unterscheidet sich dieser Fall von dem des Menschenrechtsaktivisten. Mehr noch: Die Verbindung zwischen Berta und der Gruppe ist so eng, dass jeder Angriff auf die Gruppe aus Bertas Sicht zugleich auch einen Angriff auf sie als Person darstellt. Dies ist nicht der Fall, weil ihre eigene Fortexistenz dadurch tatsächlich gefährdet wäre. Natürlich würde auch Berta die Schließung des Schachclubs überleben. Aber sie müsste ihr Leben anders ausrichten und also in diesem Sinne ein ganz anderes Leben führen als bisher.

Nun würden wir in diesem Fall natürlich noch nicht von einer kollektiven Furcht sprechen wollen, sofern Berta die Emotion nur als einzelne empfindet. Interessanter wird der Fall aber, wenn wir uns vorstellen, dass mehrere Individuen in der fraglichen Gruppe diese Emotion aufweisen, denn dann scheinen wir es mit einem Fall von kollektiver Furcht zu tun zu haben, dem zugleich auch noch eine besonders starke Identifikation der fraglichen Individuen mit der Gruppe zugrunde liegt. 
Sicherlich ist eine solche enge Identifikation mit Kollektiven wie Clubs oder Vereinen eher selten. Dennoch lassen sich davon ausgehend auch komplexere Fälle strukturell erschließen. So wirkt eine sehr starke Identifikation mit einer Gruppe etwa bei Religionsgemeinschaften oder auch Parteien schon deutlich plausibler. Diese Arten von Gruppen haben sehr viel häufiger (wenn auch keinesfalls immer) eine lebensbestimmende Funktion für ihre Mitglieder. Interessant sind auch diese Fälle, weil hier zwei Elemente gemeinsam auftreten: Das Kollektiv selbst wird als bedroht und schützenswert verstanden und die Individuen, die Furcht empfinden, identifizieren sich in besonderer Weise mit eben diesem Kollektiv.

Fälle dieser Art treten nur in Kollektiven mit relativ hoher Bindungskraft auf. Deshalb mag der Fall der Religionsgemeinschaft auch prima facie plausibler wirken als der des Schachclubs. Gleichzeitig kann die kollektive Furcht für die Mitglieder solcher Kollektive auch ein Signal dahingehend sein, dass andere das Kollektiv als ähnlich bedeutsam ansehen wie sie selbst. Dementsprechend kann eine solche Emotion die Mitglieder noch enger aneinander und an die Gruppe als Ganze binden.

Dieser Fall von Furcht ist auch interessant, weil hier die egozentrischen Aspekte individueller, existenzieller Furcht in abgewandelter Form wieder auftreten. Existenzielle, individuelle Furcht, so hatte ich im Anschluss an Martha Nussbaum betont, fokussiert das Individuum auf seine eigenen Interessen. Damit verbunden ist die Einnahme einer Perspektive auf die Welt, in der andere sowie deren Interessen nur noch eine untergeordnete Rolle spielen.

Wenn wir nun davon ausgehen, dass in manchen Fällen eine so starke Identifikation zwischen Individuen und Gruppen besteht, dass nicht mehr klar zwischen einer Gefährdung der Gruppe und einer Gefährdung des Individuums unterschieden werden kann, dann scheint es plausibel, dass ganz ähnliche strukturelle Eigenschaften auftreten. Allerdings spielt in diesem Fall eben nicht das Individuum und sein Überleben die zentrale Rolle, sondern vielmehr die Fortexistenz der Gruppe. Sie ist es, der nun die ausschließliche Aufmerksamkeit seitens der individuellen Gruppenmitglieder gewidmet wird.

Auch diese Form der Furcht kann durchaus wertvoll sein, birgt aber zugleich natürlich auch ein großes destruktives Potential. Dieses besteht, ganz ähnlich wie im ersten Fall, darin, dass Furcht dazu verleitet, ohne Rücksicht auf Verluste das eigene Interesse durchzusetzen. Allerdings handelt es sich in diesem Fall eben nicht um individuelle Interessen, sondern vielmehr um die Interessen der Gruppe. 


\section{Schluss}

Es hat sich gezeigt, dass der Ausdruck „kollektive Furcht“ kein einheitliches Phänomen bezeichnet. Vielmehr kann er mit Bezug auf zahlreiche, strukturell mitunter sehr unterschiedliche Fälle verwendet werden. Zentrale Differenzen zwischen diesen Fällen waren insbesondere im Hinblick auf das Verhältnis zwischen Individuum und Gruppe festzustellen. So zeigte sich bei der im Rahmen dieses Artikels untersuchten Spielart kollektiver Furcht, die ich als ,ichbezogene Angst“ bezeichnet habe, dass Kollektive bisweilen lediglich als ein Mittel zum Zweck verstanden werden. Eine andere Struktur wiesen hingegen die zweite und dritte betrachtete Fallgruppe auf. So zeigte sich bei der zweiten Fallgruppe, dass Furcht auch aufgrund bestimmter in einer Gruppe geteilter Anliegen zustande kommen kann, was wiederum eine sehr viel engere Verbindung zwischen Individuum und Kollektiv voraussetzt. Im dritten betrachteten Fall war diese Beziehung noch enger. Hier lag eine starke Identifikation des Individuums mit der Gruppe vor, die zu einem Verhältnis führte, das man durchaus als symbiotisch bezeichnen kann.

Wie ich betont habe, kann man schlecht über Gruppenemotionen (und insbesondere über kollektive Furcht) sprechen, ohne das destruktive Potential in den Blick zu nehmen, das diese Gefühle aufweisen. Aber auch hier muss man genau hinsehen. Es gibt nicht das eine destruktive Potential, das in allen betrachteten Fällen kollektiver Furcht vorliegt, sondern dieses ist ebenfalls stark von dem Verhältnis von Individuum und Gruppe abhängig. So zeigte sich, dass ich-bezogene Angst insbesondere problematisch ist, weil sie das Individuum auf eine rein egozentrische Perspektive festlegt, die kaum erlaubt, die Interessen anderer $\mathrm{zu}$ berücksichtigen. Anders verhielt es sich in den Fällen, in denen der kollektiven Furcht geteilte Anliegen zugrunde lagen. In diesem Fall liegen die Probleme eher in dem spezifischen Problemlösungsverhalten, das kollektive Furcht mit sich bringt. Bei der letzten betrachteten Spielart kollektiver Furcht trat hingegen aufgrund der starken Identifikation des Individuums mit dem Kollektiv die Gruppe selbst in den Vordergrund. So liegt das destruktive Potential dieser Form kollektiver Furcht meiner Analyse zufolge darin, dass hier eine rein auf die Gruppe fokussierte Perspektive eingenommen wird. Diese ähnelt der egozentrischen Perspektive dahingehend, dass anderen Interessen im schlimmsten Fall gar kein Raum mehr gegeben werden kann.

Ich habe im Rahmen dieses Artikels nur einige wenige Spielarten kollektiver Furcht in den Blick nehmen können. Eine feinkörnigere, ausführlichere Typologisierung würde sicherlich noch $\mathrm{zu}$ weiteren Differenzierungen führen. Deutlich geworden sein sollte aber auch schon vor dem Hintergrund dieser eher 
grobkörnigen Analyse, dass es kollektive Furcht simpliciter nicht gibt. Vielmehr lohnt es sich nicht zuletzt im Rahmen der auf größere gesellschaftliche Zusammenhänge abzielenden Diskussionen stets auch zu fragen, mit welcher Art von kollektiver Angst wir es denn gerade zu tun haben. Erst so lassen sich die möglichen Folgen und Gefahren, die vom Phänomen ausgehen, richtig einschätzen. Und nur dies erlaubt wiederum eine adäquate Reflexion darüber, was der richtige Umgang mit der vorliegenden kollektiven Emotion ist.

\section{Literatur}

Berninger, Anja (2017): Gefühle und Gedanken: Entwurf einer adverbialen Emotionstheorie. Münster: Mentis.

Biess, Frank (2019): Republik der Angst: Eine andere Geschichte der Bundesrepublik. Reinbek bei Hamburg: Rowohlt.

Bude, Heinz (2016): Gesellschaft der Angst, 5. Ausgabe. Hamburg: Hamburger Edition.

Corey, Robin (2006): Fear: The History of a Political Idea. Oxford: Oxford University Press.

Fredrickson, Barbara (2001): The Role of Positive Emotions in Positive Psychology: The Broaden-and-Build Theory of Positive Emotions. American Psychologist 56 (3), 218-226.

Helm, Bennett W. (2001): Emotional Reason: Deliberation, Motivation, and the Nature of Value. Cambridge: Cambridge University Press.

Helm, Bennett W. (2008): Plural Agents. Noûs 42 (1), 17-49.

Helm, Bennett W. (2009): Emotions as Evaluative Feelings. Emotion Review 1 (3), 248-255.

Hobbes, Thomas (1996): Leviathan. Hamburg: Felix Meiner.

Huebner, Bryce (2011): Genuinely collective emotions. European Journal for Philosophy of Science 1 (1), 89-118.

Isen, Alice M., Kimberly A. Daubman, \& Gary P. Nowicki (1987): Positive affect facilitates creative problem solving. Journal of Personality and Social Psychology 52, 1122-1131.

Klovert, Heike (2019): Brandbrief zu EU-Austritt: Britische Universitäten fürchten ungeregelten Brexit. Spiegel Online, https://www.spiegel.de/lebenundlernen/uni/no-deal-brexitbritische-hochschulen-warnen-vor-ungeregeltem-austritt-a-1246387.html. (7. Oktober, 2019.)

List, Christian \& Philip Pettit (2011): Group Agency. The Possibility, Design, and Status of Corporate Agents. Oxford: Oxford University Press.

Nachtwey, Oliver (2017): Die Abstiegsgesellschaft: Über das Aufbegehren in der regressiven Moderne, 6. Ausgabe (Edition Suhrkamp 2682). Berlin: Suhrkamp.

Nussbaum, Martha C. (2001): Upheavals of Thought: The Intelligence of Emotions. Cambridge: Cambridge University Press.

Nussbaum, Martha C. (2019): Königreich der Angst: Gedanken zur aktuellen politischen Krise. Darmstadt: Wissenschaftliche Buchgesellschaft.

Quigley, Leanne, Andrea L. Nelson, Jonathan Carriere, Daniel Smilek \& Christine Purdon (2012): The effects of trait and state anxiety on attention to emotional images: An eye-tracking study. Cognition and Emotion 26 (8), 1390-1411. 
Roberts, Robert (2003): Emotions: An Essay in Aid of Moral Psychology. Cambridge: Cambridge University Press.

Robinson, Jenefer (2005): Deeper than Reason: Emotion and its Role in Literature, Music, and Art. Oxford: Oxford University Press.

Salmela, Mikko (2014): The Functions of Collective Emotions in Social Groups. In: Anita Konzelmann Ziv \& Hans B. Schmid (Hrsg.), Institutions, Emotions, and Group Agents, 159-176. Dordrecht: Springer Netherlands.

Solomon, Robert (1993): The Passions: Emotions and the Meaning of Life. Indianapolis/Cambridge: Hackett.

Tietjen, Ruth R. (2019): Am Abgrund: Philosophische Theorie der Angst und Übung in philosophischer Freiheit. Paderborn: Mentis.

von Scheve, Christian \& Sven Ismer (2013): Towards a Theory of Collective Emotions. Emotion Review 5 (4), 406-413. 
